\title{
Epigenetic silencing of miR-181c by DNA methylation in glioblastoma cell lines
}

\author{
Erandi Ayala-Ortega, Rodrigo Arzate-Mejía, Rosario Pérez-Molina, Edgar González-Buendía, Karin Meier,
} Georgina Guerrero and Félix Recillas-Targa*

\begin{abstract}
Background: Post-transcriptional regulation by microRNAs is recognized as one of the major pathways for the control of cellular homeostasis. Less well understood is the transcriptional and epigenetic regulation of genes encoding microRNAs. In the present study we addressed the epigenetic regulation of the miR-181C in normal and malignant brain cells.
\end{abstract}

Methods: To explore the epigenetic regulation of the miR-181C we evaluated its expression using RT-qPCR and the in vivo binding of the CCCTC-binding factor (CTCF) to its regulatory region in different glioblastoma cell lines. DNA methylation survey, chromatin immunoprecipitation and RNA interference assays were used to assess the role of CTCF in the miR-181C epigenetic silencing.

Results: We found that miR-181C is downregulated in glioblastoma cell lines, as compared to normal brain tissues. Loss of expression correlated with a notorious gain of DNA methylation at the miR-181c promoter region and the dissociation of the multifunctional nuclear factor CTCF. Taking advantage of the genomic distribution of CTCF in different cell types we propose that CTCF has a local and cell type specific regulatory role over the miR-181C and not an architectural one through chromatin loop formation. This is supported by the depletion of CTCF in glioblastoma cells affecting the expression levels of NOTCH2 as a target of miR-181C. Conclusion: Together, our results point to the epigenetic role of CTCF in the regulation of microRNAs implicated in tumorigenesis.

Keywords: Glioblastoma cells, CCCTC-binding factor (CTCF), DNA methylation, RNA interference, Epigenetics

\section{Background}

MicroRNAs (miRNAs) are small non-coding RNAs that participate in the control of many cellular processes such as stress response, cell differentiation, cell-cycle regulation, stem cell biology, apoptosis among many others [1]. MicroRNAs exert their regulatory effect post-transcriptionally by inducing RNA degradation or translation inhibition, and their expression can be deregulated in cancer by genetic and epigenetic mechanisms [2-4]. MicroRNAs can also affect gene expression of many genes by direct regulation of the epigenetic machinery. For example, microRNAs like miR-101, miR-205 and miR-26a regulate chromatin modifiers in cancer such as the Polycomb associated

\footnotetext{
* Correspondence: frecilla@ifc.unam.mx

Instituto de Fisiología Celular, Departamento de Genética Molecular,

Universidad Nacional Autónoma de México, Ciudad de México, México
}

histone methyltransferase EZH2 [2, 3]. The DNA methylation maintenance enzyme Dnmt1 is regulated in different cell- types by the miR-126 and miR-152, as well as the de novo methyltransferases Dnmt3a and Dnmt3b by the miR-29 family members miR-29a, $-29 b$ and $-29 c$ [5]. Overexpression of $m i R-29 a,-29 b$ and $-29 c$ cause abnormal downregulation of the Dnmt3a and Dnmt3b, which is associated with development of lung cancer and acute myeloid leukemia [6,7].

DNA methylation can regulate microRNAs gene expression in cancer [8]. In particular, repression of gene expression by DNA methylation of promoter associated $\mathrm{CpG}$ islands has been reported for several microRNAs in glioblastoma cells like miR-211, miR-204, $m i R-145, m i R-137$ among others [9-12]. For example, miR-145 was shown to be downregulated in glioblastoma cells and low expression of $m i R-145$ was found to 
be correlated with poor prognosis in patients [11]. Overexpression of $m i R-145$ reduced cell proliferation, migration and invasion in glioblastoma cells by suppressing SOX9 and ADD3 [13]. Thus, DNA methylation of CpGrich microRNAs promoters in glioblastoma cells seems to be an important process for tumour development and maintenance.

CTCF is a ubiquitous, highly-conserved 11-zinc finger nuclear protein $[14,15]$, which is subjected to different post-translational modifications $[16,17]$ and has been implicated in a broad range of functions including higher-order chromatin organization by favoring interand intra-chromosomal interactions [18-20]. The combinatorial usage of different zinc-fingers confers CTCF the capacity to bind complex sequences, interact with other proteins and with ncRNAs [14, 21-23]. CTCF is also important to maintain, CpG-rich promoter regions of tumour suppressor genes, like BRCA1, retinoblastoma, and others, in an unmethylated state [24, 25]. Importantly, DNA methylation can affect CTCF binding in part because of the presence of CpGs in the CTCF binding motif [26]. For example, increased methylation at the promoter of the brain-derived neurotrophic factor $(B D N F)$ triggered the dissociation of CTCF which resulted in gene silencing [27]. In fact $41 \%$ of cell-type specific CTCF binding sites show differential DNA methylation [28].

In addition, several reports have implicated CTCF in the regulation of microRNAs expression [29]. MiR-125b expression is decreased in breast cancer, partly, through CTCF dissociation from its promoter region [30]. In addition, ER $\alpha$ positive breast cancer cells overexpress miR-375 concomitantly with promoter DNA hypermethylation and CTCF depletion [31]. Furthermore, CTCF and pluripotency maintenance factors are depleted in the miR-290 regulatory region in differentiated embryonic stem cells, together with increased DNA methylation and deposition of the repressive histone mark H3K27me3 [32].

The miR-181c is a member of the miR-181 family of microRNAs involved in the development of glioblastoma multiforme $(\mathrm{GBM})$, which is one of the most frequent and malignant primary brain tumours [33, 34]. MiR-181c is downregulated in GBM, and its expression levels correlate with tumour progression, suggesting that its epigenetic regulation could be affected [33]. In contrast, miR-181c is overexpressed in gastric cancer, skin basal cell carcinoma, and in osteosarcomas [35-37].

Here we explored the epigenetic regulatory processes responsible for the deregulation of $m i R-181 c$ in glioblastoma cells; in particular, we asked whether the nuclear factor CTCF participates in its epigenetic regulation. We first confirmed that miR-181c is differentially expressed in glioblastoma cell lines. We analyzed ChIP-seq data sets from different cell-types and identified a DNA region located in the $5^{\prime}$ non-coding region of the $m i R$ $181 \mathrm{c}$ enriched in histone marks characteristic of promoter regions. We confirmed binding of CTCF to the promoter region of $m i R-181 \mathrm{c}$ in the glioblastoma cell line U87MG and K562 cells. In contrast, CTCF does not bind the promoter region of the aggressive glioblastoma cell line T98G. Absence of CTCF correlates with gain of DNA methylation and miR-181c downregulation. Furthermore, we show that depletion of CTCF in glioblastoma cells affects the expression levels of NOTCH2 a target of miR-181c. Together, these results implicate CTCF and DNA methylation in the epigenetic regulation of $m i R-181 c$ in cancer cells.

\section{Methods}

\section{Cell culture}

K562 human erythroleukemic cells were cultured in ISCOVE medium (Invitrogen). K562 cells (K562 ATCC CCL-243 $3^{\mathrm{mm}}$ ) were provided by Gary Felsenfeld (National Institutes of Health, Bethesda, Maryland, US); human glioblastoma-astrocytoma grade IV U87MG cells (U87MG ATCC $^{\circ}$ HTB- $\left.4^{\mathrm{mm}}\right)$, human glioblastoma multiforme T98G cells (T98G ATCC ${ }^{\oplus}$ CRL-1690 ${ }^{\text {max }}$ ) and human acute T cell leukemia Jurkat cells (Jurkat ATCC ${ }^{\odot}$ TIB-152 $2^{\mathrm{mm}}$ ) were cultured in RPMI-1640 medium (Invitrogen); all media contained $10 \%(\mathrm{v} / \mathrm{v})$ fetal bovine serum (FBS) and $1 \%$ penicillin/streptomycin. T98G, U87MG and Jurkat cells were provided by Manel Esteller (Centro Nacional de Investigaciones Oncológicas (CNIO) and Cancer Epigenetics and Biology Program (PEBC), Spain). All cell lines were purchased from the American Type Culture Collection (Manassas, VA) and were previously authenticated by STR profiling. Cells were maintained at $37^{\circ} \mathrm{C}$ in a humidified $5 \% \mathrm{CO}_{2}$-containing atmosphere. $\mathrm{Hu}$ man lymphocytes were obtained from peripheral blood of a healthy donor, isolated with Ficoll-Paque Plus (Amersham) following the manufacturer's instructions. Written informed consent was obtained from this healthy donor.

\section{Quantitative real time PCR}

Total RNA from Human Hypothalamus and Orbital Frontal Cortex were purchased from Ambion (First Choice ${ }^{\circ}$ Total RNA AM6786 and AM6864). Total RNA was extracted from lymphocytes, K562, Jurkat, U87MG and T98G cells with TRIzol Reagent (Invitrogen) according to manufacturer's instructions. RNAs were treated with DNase I (RQ1, Promega) followed by Random Primer cDNA generation from $1 \mu \mathrm{g}$ DNase I treated RNA (Reverse Transcription System, Promega). Real-Time quantitative PCR (qPCR) was carried out with SYBR Green (Sigma) and specific primers for primiR-181c (Forward: 5' -CCCATCTCAGCCTCCTAAGT-3' and Reverse: 
5'-GACCAACCTGAGCAACATAG-3'), NOTCH2 (Forward: 5'-CCTTCCACTGTGAGTGTCTGA-3' and Reverse: 5' - AGGTAGCATCATTCTGGCAGG-3') and GAPDH as an endogenous normalization control (Forward: 5'-CCACTCCTCCACCTTTGAC-3' and Reverse: $5^{\prime}$-ACCCTGTTGCTGTAGCCA-3'). In order to analyze miR-181c mature transcript levels, first strand cDNA was generated using Taqman ${ }^{\circ}$ MicroRNA Reverse Transcription Assay (Applied Biosystems) with specific primers provided by the manufacturer and U6 RNA was used as an endogenous normalization control. MiR-181c mature transcript levels were measured with Taqman ${ }^{\circ}$ MicroRNA Assay primers (Applied Biosystems). The qPCR reactions were carried out in the StepOne detection system (Applied Biosystems) at $95{ }^{\circ} \mathrm{C}$ for 2 min, followed by 40 two-step cycles of $95{ }^{\circ} \mathrm{C}$ for $30 \mathrm{~s}$ and $60{ }^{\circ} \mathrm{C}$ for $45 \mathrm{~s}$, triplicates were made for each sample. Relative RNA levels were calculated using the comparative $\Delta \Delta \mathrm{Ct}$ method. Significant differences on gene expression were evaluated by a $t$-Student test.

\section{DNA sodium bisulfite conversion}

Genomic DNA was extracted from indicated cells by phenol-chloroform technique, and $1.5 \mu \mathrm{g}$ were cut with HindIII previous to bisulfite conversion. Bisulfite conversion was performed as described previously [38]. Specific primers for converted promoter region were used to generate PCR product (Forward: 5'-GTTTTAGATAGA GGGGTGGG-3' and Reverse: 5' - CAATCCTCAAAAAA CCCAACTC-3'). PCR products were cloned in pGEMTeasy (Promega) followed by sequencing with Sp6 primer. Culture recuperation after transformation for plasmid enrichment was carried out at $30{ }^{\circ} \mathrm{C}$ to avoid recombination as much as possible.

\section{Chromatin immunoprecipitation assay}

The ChIP assay was performed as previously reported with $4 \mu \mathrm{g}$ of antibody against CTCF (Millipore 07-729) [38]. Immunoprecipitated DNA was evaluated by PCR using specific amplification primers for CTCF downstream (Forward: 5'-GTCTCAACTTCTGGGCTCC-3' and Reverse: 5'-GAAGAGAAATAGGCGGTGG-3'), Upstream (Forward: 5' -CTCCCATCTCAGCCTCCTA$3^{\prime}$ and Reverse: 5' -CAAGCCAAGCAGTGACGAC-3') regions, and Igf2/H19 Differential Methylation Region (DMR) as a positive control (Forward: 5'-CAGGCTCC CCCAAAATCTA-3' and Reverse: 5'-GGGAACATAG AGAAAGAGG-3').

\section{CTCF knockdown with lentivirus expressing shRNAi}

CTCF knockdown was performed essentially as described [39]. HEK293FT cells were used to produce pLL3.7 control and CTCF shRNAi (5'-GGACAGTGTT TGACAACTAA-3') lentiviruses with generation III packaging vectors. pLL3.7 and shRNAi CTCF plasmid were kindly provided by Joaquín Espinosa [39]. For the tetracycline inducible system the pTRIPZ lentiviral vector (Open Systems) was used with shRNAi (5'-AGGAC AGTGTTGACAACTA-3') targeting CTCF. U87MG cells were transduced with virus for $8 \mathrm{~h}$ in the presence of polybrene $(8 \mu \mathrm{g} / \mathrm{ml}$; Sigma). Cultures were then selected for 3-4 days with puromycin $(5 \mu \mathrm{g} / \mathrm{ml}$; Sigma) and then harvested for the experiments detailed in this article. Doxycycline induction was carried out with $2 \mu \mathrm{g} / \mathrm{ml}$ for $72 \mathrm{~h}$, and cells were harvested for the corresponding experiments.

\section{Bioinformatic analysis}

All ChIP-seq and RRBS data was downloaded from the Analysis/Data hub from the ENCODE project (https:// genome.ucsc.edu/ENCODE/analysis.html) and displayed on the IGV genome browser (https://www.broadinstitute.org/igv/node/250). CTCF Motif analysis was performed with JASPAR using the human motif as query (http://jaspar.genereg.net/). CpG islands were downloaded from the UCSC genome browser hg19 (https://genome.ucsc.edu/). In situ $\mathrm{Hi}-\mathrm{C}$ data from GM12878 cell line at $5 \mathrm{~kb}$ resolution was analyzed by using the JuiceBox software (http://www.aidenlab.org/juicebox/).

\section{Results \\ Differential expression of miR-181c in brain and human glioblastoma cells}

The human miR-181c is frequently downregulated in Glioblastoma Multiforme (GBM) and its downregulation has been linked to tumour progression [33]. However, the mechanisms controlling its expression are unknown. To identify the regulatory region of miR-181c we analyzed ChIP-seq data for the promoter-associated histone marks H3K4me3 and H3K27ac generated by ENCODE in the erythroleukemic K562 cell line. We identified a DNA region occupied by H3K4me3 and H3K27ac located $2 \mathrm{k}$ bases $(\mathrm{kb})$ upstream of the sequence corresponding to the mature miR-181c (Fig. 1a and Additional file 1: Figure S1). This DNA region was previously reported to act as a promoter of $m i R-181 c$ [40]. The identified region overlaps with a $\mathrm{CpG}$ Island of $0.5 \mathrm{~kb}$ containing a CTCF binding motif. Indeed, ChIPseq data shows that CTCF binds the promoter region (Fig. 1a). Since CTCF binds to a CpG rich region in the promoter of miR-181c we hypothesized that DNA methylation and CTCF could be critical regulators of miR-181c expression in glioblastoma.

As a first step to uncover the mechanisms controlling miR-181c we first evaluated transcript levels for the pri$m i R-181 c$ and mature miR-181c by RT-qPCR in two glioblastoma cell lines T98G and U87MG, erythroleukemic K562 cells, lymphoblastic Jurkat cells, peripheral 


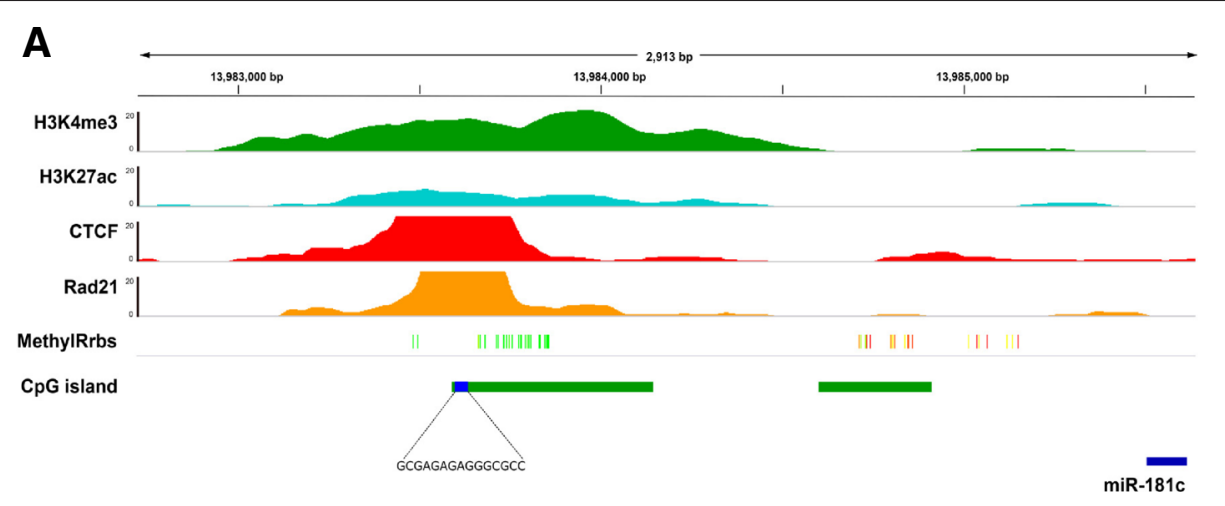

B

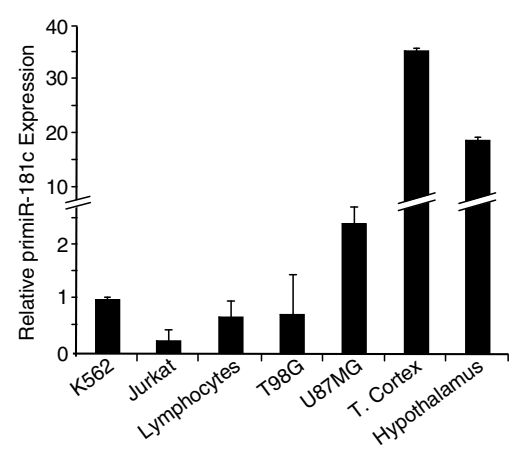

C

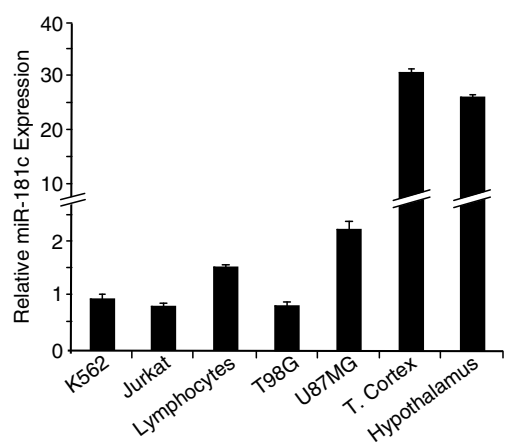

Fig. 1 CTCF binds to the promoter of miR-181C. a IGV genome browser screenshot for ChIP-seq data of H3K4me3, H3K27ac, CTCF and Rad21 from K562 cells and Reduced Representation Bisulfite Sequencing (RRBS) data from the same cell line. Green bars, 0 \% molecules sequenced are methylated; Yellow bars, $50 \%$ molecules are methylated; Red bars, $100 \%$ molecules sequenced are methylated. CTCF binding motif with the highest score is shown with reference to one CpG island. The region depicted is chr19:13,982,729-13,985,645. Data was downloaded from the Analysis/Data hub by the ENCODE project. b primiR-181c expression levels in different cells measured by RT-qPCR with SYBR Green. c miR-181c expression levels in different cells measured by Taqman assay

blood lymphocytes, frontal cortex and hypothalamusderived primary cells (Fig. $1 \mathrm{~b}$ and c). The highest level of expression of the primiR-181c and the mature miR-181c transcript were found in cells from the frontal cortex and hypothalamus, which is consistent with previous reports showing that $m i R-181 c$, is mainly expressed in brain cells in human, mouse and rat (Expression Atlas EMBL-EBI). Intermediate levels of expression were found in U87MG glioblastoma cell line, and very low levels in the aggressive glioblastoma cell line T98G and the rest of the analyzed cells (Fig. $1 \mathrm{~b}$ and c). Thus miR$181 \mathrm{c}$ is expressed at low levels in glioblastoma cell lines as compared with brain primary cells.

\section{Low levels of miR-181c in glioblastoma cells correlate with DNA hypermethylation of its promoter region}

In order to characterize the DNA methylation profile of the $m i R-181 c$ promoter region we performed DNA bisulfite conversion coupled to sequencing in T98G and U87MG glioblastoma cell lines, K562 cells and primary lymphocytes (Fig. 2a). The highest DNA methylation levels of the miR-181c promoter, with $86 \%$ of methylated CpGs, were found in T98G (Fig. 2a). Intermediate
DNA methylation, with $47 \%$ of methylated CpGs, was found in U87MG. The promoter region was almost unmethylated, with 0.6 and $5 \%$ of methylated CpGs, in K562 cells and lymphocytes, respectively (Fig. 2a). Hypermethylation of the miR-181c promoter region correlates with the low level of transcript detected in T98G cells. A 2-fold increase in the expression of the primiR-181c in U87MG cells, as compared with T98G cells, correlates with a $50 \%$ reduction in the methylation of the promoter. Low levels of expression of $m i R$ $181 \mathrm{c}$ and primiR-181c do not correlate with absence of DNA methylation in K562 cells and lymphocytes. This is probably due to the tissue-specific expression of the miR-181c and the absence in K562 cell of a particular set of transcription factors and co-factors needed for miR-181c gene transcription.

DNA hypermethylation of promoter regions of microRNAs has been linked to transcriptional repression [8]. To determine if the hypermethylation of the promoter region of miR-181c cells promotes transcriptional repression, we treated T98G cells with the DNA methylation inhibitor 5-aza-2'-deoxycytidine (5-azadC) for $72 \mathrm{~h}$ and then analyzed the expression of the primary 


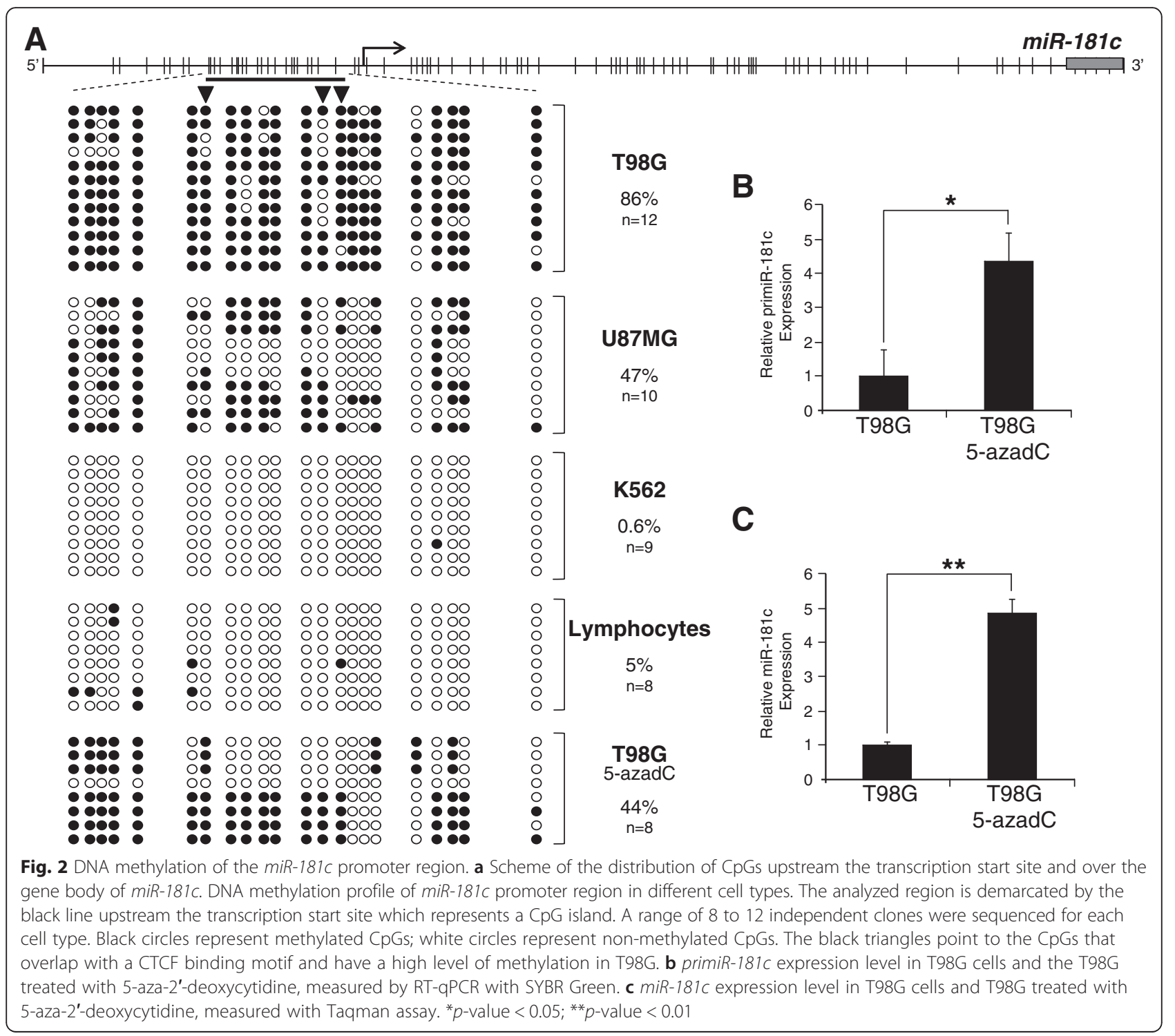

primiRNA-181c and mature miR-181c. To confirm these results we performed DNA bisulfite genomic DNA conversion and sequencing in T98G genomic DNA previously treated with 5 -azadC. More than $40 \%$ of the CpGs are demethylated, and of note, the CTCF binding site seems preferentially unmethylated (Fig. 2a). In line with this, we found that the expression levels of miR-181c increased after treatment (Fig. 2b and c), suggesting silencing of the $m i R-181 c$ by DNA methylation in glioblastoma cells.

Interaction of CTCF with the regulatory region of miR-181c

The region of the miR-181c occupied by CTCF in K562 cells as indicated by ENCODE data, spans the $7^{\text {th }}, 13^{\text {th }}$ and $14^{\text {th }}$ CpGs of the analyzed CpG-island (Fig. 2a). Importantly, methylation levels of these CpGs are different between T98G and U87MG cells (Fig. 2a arrowheads). In particular the $7^{\text {th }}$ and $14^{\text {th }}$ CpGs show a 45 and $61.6 \%$ increase in DNA methylation, in T98G as compared to U87MG, opening the possibility that DNA methylation may affect CTCF binding in this region. Analysis of ChIP-seq data from ENCODE shows interaction of CTCF with the promoter region of miR-181c in 23 of the 47 cell lines analyzed, suggesting cell-type specific binding of CTCF to this region. However, if CTCF interacts with the miR-181c promoter in the glioblastoma cell lines used in this study is not known (Additional file 1: Figure S1 and Table S1). To determine if CTCF interacts with the $m i R-181 c$ promoter in T98G and U87MG cells we performed chromatin immunoprecipitation (ChIP) (Fig. 3). As a positive control we looked at CTCF enrichment on the human Igf2/H19 Differential Methylation Region (DMR) in K562 cells 


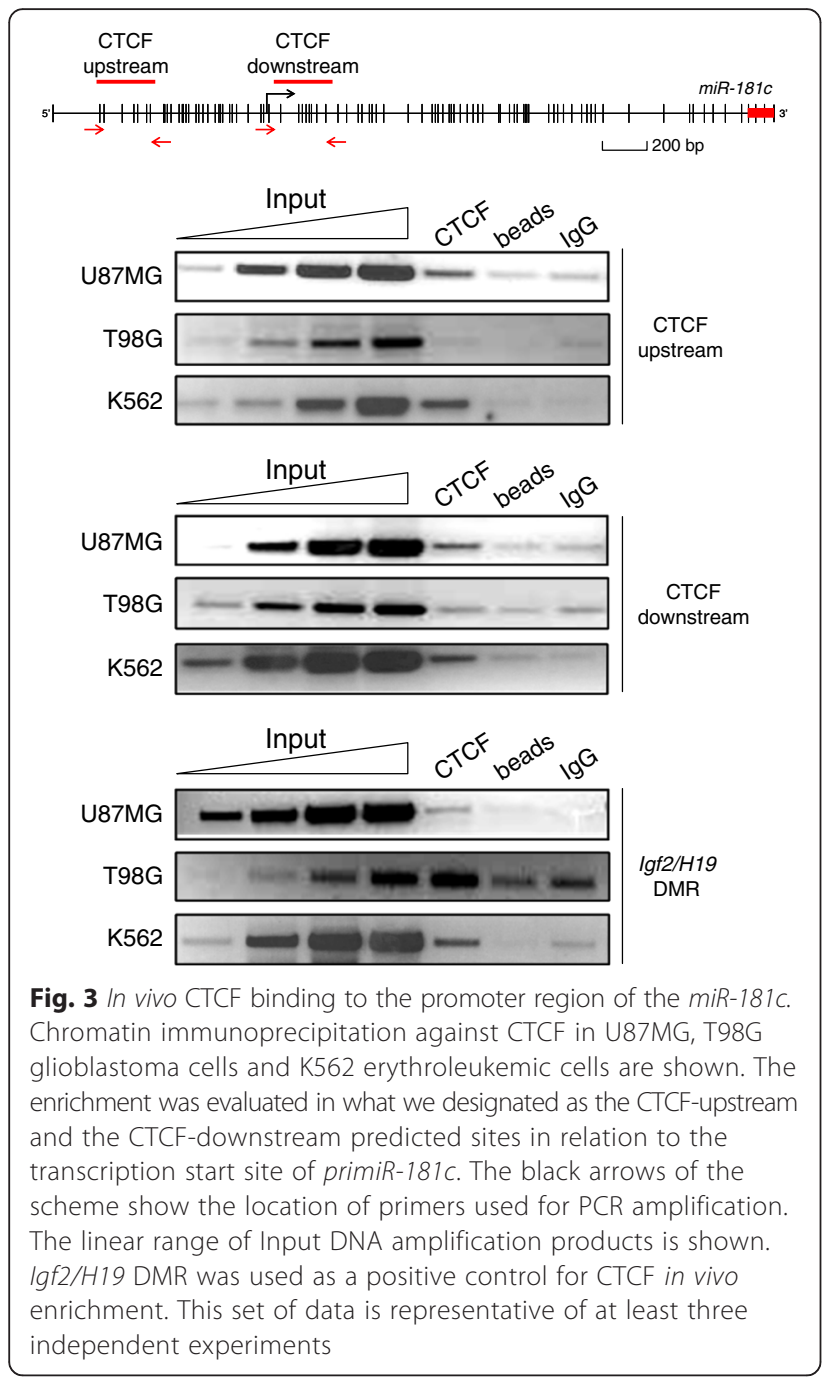

$[26,41]$. We found that CTCF interacts with the miR181c promoter in U87MG, but not in T98G cells (Fig. 3). Motif analysis suggests that additional CTCF binding sites are present over the gene body of $m i R$ $181 \mathrm{c}$, however, they are not bound by CTCF in U87MG, T98G and K562 cells, as revealed by ChIP assays (Additional file 1: Figure S2).

The interaction of CTCF with the miR-181c promoter correlates with moderate gene expression in U87MG cells. Absence of CTCF interaction with the miR-181c promoter correlates with DNA hypermethylation and very low expression levels in T98G cells. Thus, CTCF may be associated with expression regulation and protection against DNA methylation of the miR-181c promoter in U87MG cells.

\section{CTCF occupancy correlates with miR-181c expression}

To further characterize the contribution of CTCF to miR181c regulation, CTCF was knocked down in U87MG cells by transduction with a doxycycline inducible lentivirus containing small-hairpin interference RNA (shRNAi) against CTCF (Fig. 4 and Additional file 1: Figure S3). Cells were treated with doxycycline or vehicle for $72 \mathrm{~h}$ and the expression levels of primiR-181c and mature miR-181c were assessed by RT-qPCR. MiR-181c was significantly downregulated upon CTCF knockdown (Fig. 4b and c). Doxycycline withdrawal for two weeks resulted in upregulation of miR-181c (Fig. 4b and c). This data suggests that CTCF promotes the expression of miR-181c in U87MG cells.

CTCF depletion results in increased promoter methylation and decreased expression of $m i R-181 \mathrm{C}$

To test the function of CTCF in the protection against DNA methylation of the miR-181c promoter we knocked down CTCF in U87MG cells and assessed the level of DNA methylation (Fig. 5a). After 5 days of transduction with the CTCF shRNAi we observed a $20 \%$ increase in the DNA methylation level of the $\mathrm{mi} R-181 \mathrm{c}$ promoter region (Fig. 5b). This increase in DNA methylation was accompanied with a reduction of primiR-181c and miR181c (Fig. 5c and d). These results suggest that CTCF could protect the miR-181c promoter from DNA methylation in U87MG cells.

\section{miR-181C is flanked by two chromatin loops in GM12878} cells

The three-dimensional organization of the genome is critical to establish proper programs of gene expression through the formation of chromatin loops that bring together distal regulatory regions [42]. CTCF is a key mediator of chromatin looping, and novel techniques like in situ $\mathrm{Hi}-\mathrm{C}$ coupled with deep sequencing allow the identification of all long-range chromatin interactions in a given cell-type [43]. To gain insight on whether the CTCF binding site in the promoter region of $m i R-181 c$ is implicated in loop formation we took advantage of published data of high resolution in situ $\mathrm{Hi}-\mathrm{C}$ generated in GM12878 cells [43]. The in situ Hi-C data set represents the highest resolution $(1 \mathrm{~kb})$ map of chromatin interactions ever published and identified chromatin loops at a genome wide scale. In situ Hi-C data for GM12878 cells suggest the presence of two chromatin loops (100 kb and $200 \mathrm{~kb}$ in size, respectively) flanking the miR-181c locus and part of the Nanos3 locus (Fig. 6a). The anchor sites for chromatin loops frequently overlap with binding sites for CTCF in convergent orientation (92\%) [43]. In fact, the anchor sites for the two chromatin loops flanking the miR-181c locus correspond to constitutive binding sites for CTCF in convergent orientation (Fig. 6b). In contrast, the CTCF binding site on the promoter region of $m i R-181 c$, which is occupied only in a subset of cell lines, is not involved in chromatin looping (Fig. 6b). Thus, the CTCF binding site in the 


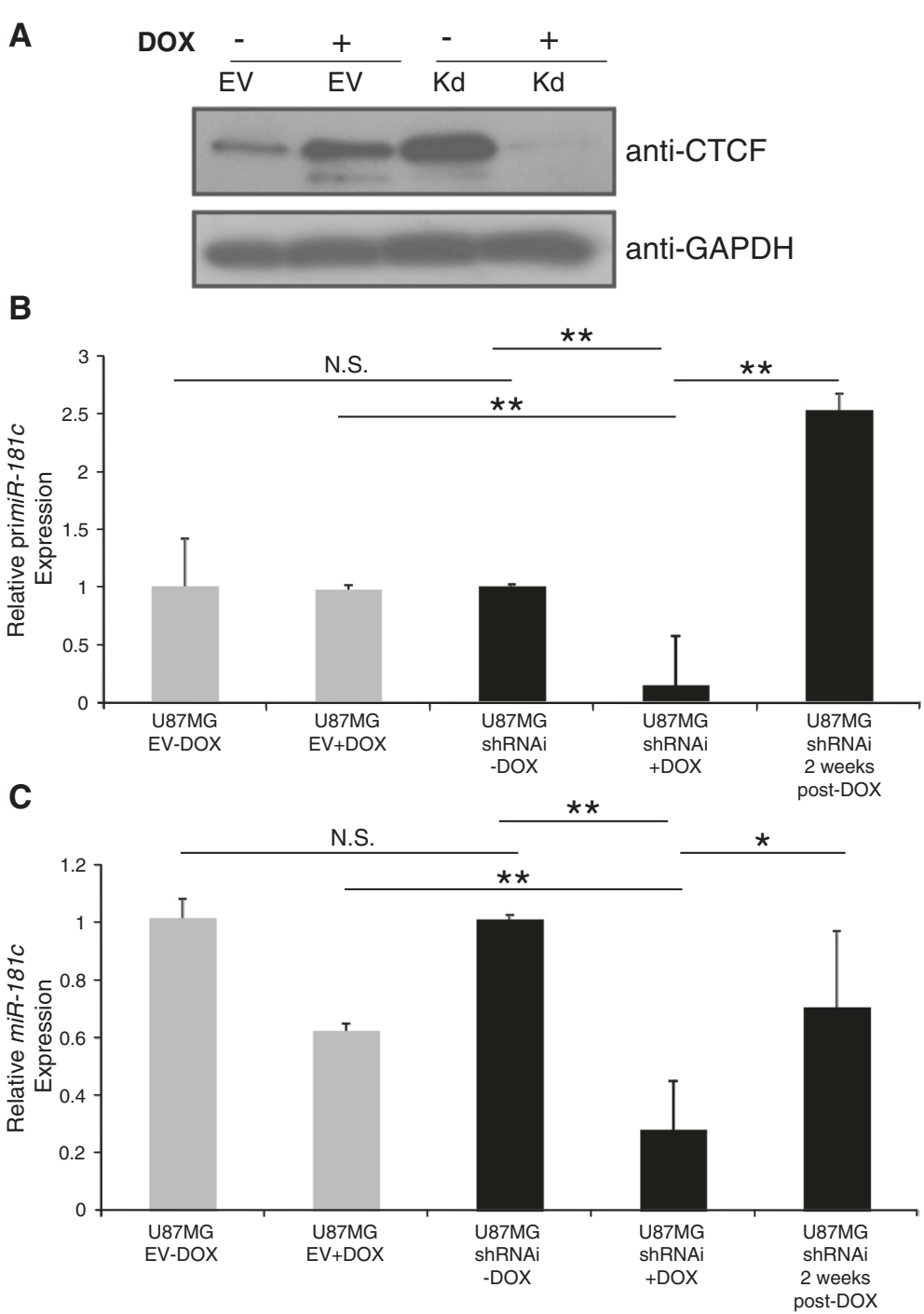

Fig. 4 Inducible knockdown of human CTCF in U87MG glioblastoma cell line affects miR-181c expression. a Western blot shows CTCF protein levels in U87MG cells transduced with an inducible Empty Vector (EV) with and without Doxycycline (DOX) induction as controls. U87MG cells were also transduced with an inducible shRNAi against CTCF without (-DOX) and with (+DOX) Doxycycline (DOX). b primiR-181c expression levels in cell pools containing the inducible shRNAi against CTCF. No treatment (U87MG/shRNAi), treatment (U87MG/shRNAi/+DOX) and 2 weeks after Doxycycline deprivation (U87MG/shRNAi/2 weeks/post-DOX) were measured by RT-qPCR with SYBR Green. Empty vector controls are shown (U87MG/EV-DOX and U87MG/EV + DOX). N.S., not significant. c The miR-181c expression levels were evaluated under the same experimental conditions as in (b) using the Taqman assay. ${ }^{*} p$-value $<0.05$ and ${ }^{* *} p$-value $<0.01$

promoter region of $m i R-181 c$ protects against DNA methylation, and we speculate that this particular site does not participate in chromatin looping.

\section{Depletion of CTCF in glioblastoma cells affects the expression levels of NOTCH2}

Our results suggest that CTCF participates in the transcriptional regulation of $m i R-181 \mathrm{c}$ by protecting its promoter against silencing by DNA methylation in U87MG cells. Therefore, we asked if reduced levels of CTCF could affect the transcript levels of $m i R-181 c$ targets like NOTCH2 [34]. We infected the glioblastoma U87MG cell line with a lentivirus expressing a doxycycline inducible shRNAi against human CTCF. Quantitative RT-PCR of NOTCH2 was performed after 3 and 30 days of induction with doxycycline. Knockdown of CTCF at 3 days after induction results in an increase of NOTCH2 mRNA (Fig. 7a). This trend is more evident with cells that have been on doxycycline during 30 days. Therefore CTCF loss causes the epigenetic silencing of miR-181c by DNA methylation and the inability of the miR-181c to diminish the 


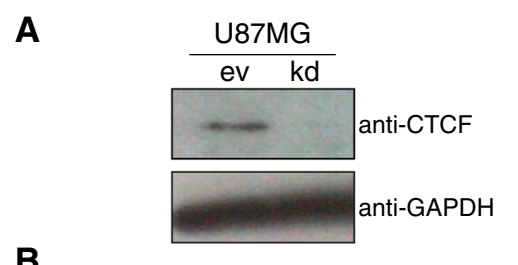

B
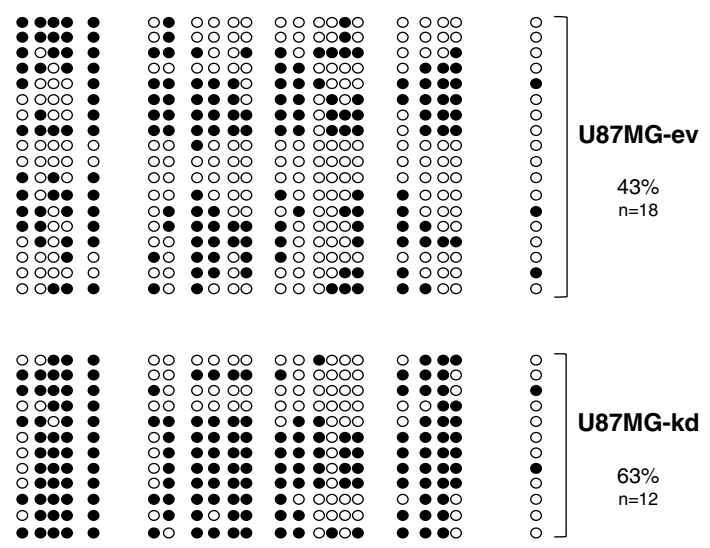
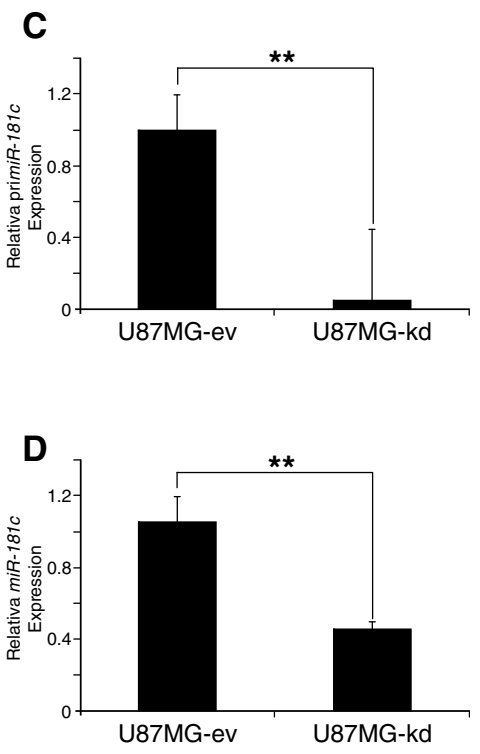

Fig. $\mathbf{5}$ CTCF depletion results in increased promoter methylation and decreased expression of miR-181c. a Western-blot showing the levels of CTCF protein in cells infected by a lentiviral system expressing a shRNAi against CTCF. $\mathbf{b}$ Comparative DNA methylation analysis of the miR-181C promoter region. The percentage of methylated CpGs is shown for knockdown cells (U87MG-kd) and control cells (U87MG-ev). Black circles correspond to methylated CpGs and white circles to unmethylated CpGs. c primiR-181c expression levels measured by RT-qPCR with SYBR Green. d miR-181c expression levels measured by Taqman Assay. ${ }^{* *} p$-value $<0.01$

levels of NOTCH2 transcripts in glioblastoma U87MG cells (Fig. 7b).

\section{Discussion}

Cancer is a multistep disease that includes many interdependent components at the cellular level [44]. There are also molecular components that include genotypic abnormalities but more recently epigenotypic deregulation [45]. In particular, and based on the relevance of the post-transcriptional regulatory function of microRNAs over different types of genes we studied here how epigenetic regulatory processes can dysregulate microRNAs transcription in cancer. We asked how a microRNA, the miR-181c, involved in the regulation of brain specific genes can be epigenetically deregulated in glioblastoma cell lines, one of the more frequently occurring primary malignant brain tumours. We focused on the glioblastoma cell lines, T98G and U87MG, were the miR-181c is downregulated in comparison to normal brain tissues. This microRNA loss of gene expression correlated with a strong gain of DNA methylation in the $m i R-181 c$ promoter region. Importantly, this aberrant DNA hypermethylation apparently interferes with the binding of the chromatin associated CTCF nuclear factor. CTCF depletion confirmed a gain of DNA methylation in U87MG cells supporting a previously reported protective role of CTCF in tumour suppressor genes [25]. Finally, CTCF knockdown induces the upregulation of NOTCH2 a target of miR-181c.

Concerning the transcriptional regulation of microRNAs an important sub-group is annotated as intergenic, but others are intronic and/or exonic, either in sense or antisense orientations presenting a more complex regulatory context. Genetic disruption of microRNAs has been documented in cancer, but there are some evidences that suggest that epigenetic alterations can be one of the major mechanisms for microRNA deregulation in cancer and other diseases [2]. There is a growing list of microRNAs that are subjected to epigenetic abnormal influence, including gain or loss of DNA methylation, histone covalent modifications, and more recently, the topological organization of the genome (see below). For example, it is well documented how members of the miR-34 family are involved in cancer through cell cycle arrest, cell invasion, apoptosis or even cancer metastasis [2]. These microRNAs are mainly silenced by DNA methylation of their promoter regions. Concerning the role of CTCF in microRNAs, a recent report showed that the miR-125b1 is aberrantly silenced by DNA methylation in breast cancer cells [30]. In such context, CTCF binding to the promoter region of the miR-125b1 is disrupted and a gain in the repressive histone modification $\mathrm{H} 3 \mathrm{~K} 9 \mathrm{me} 3$ and $\mathrm{H} 3 \mathrm{~K} 27 \mathrm{me} 3$ is detected in cancer 


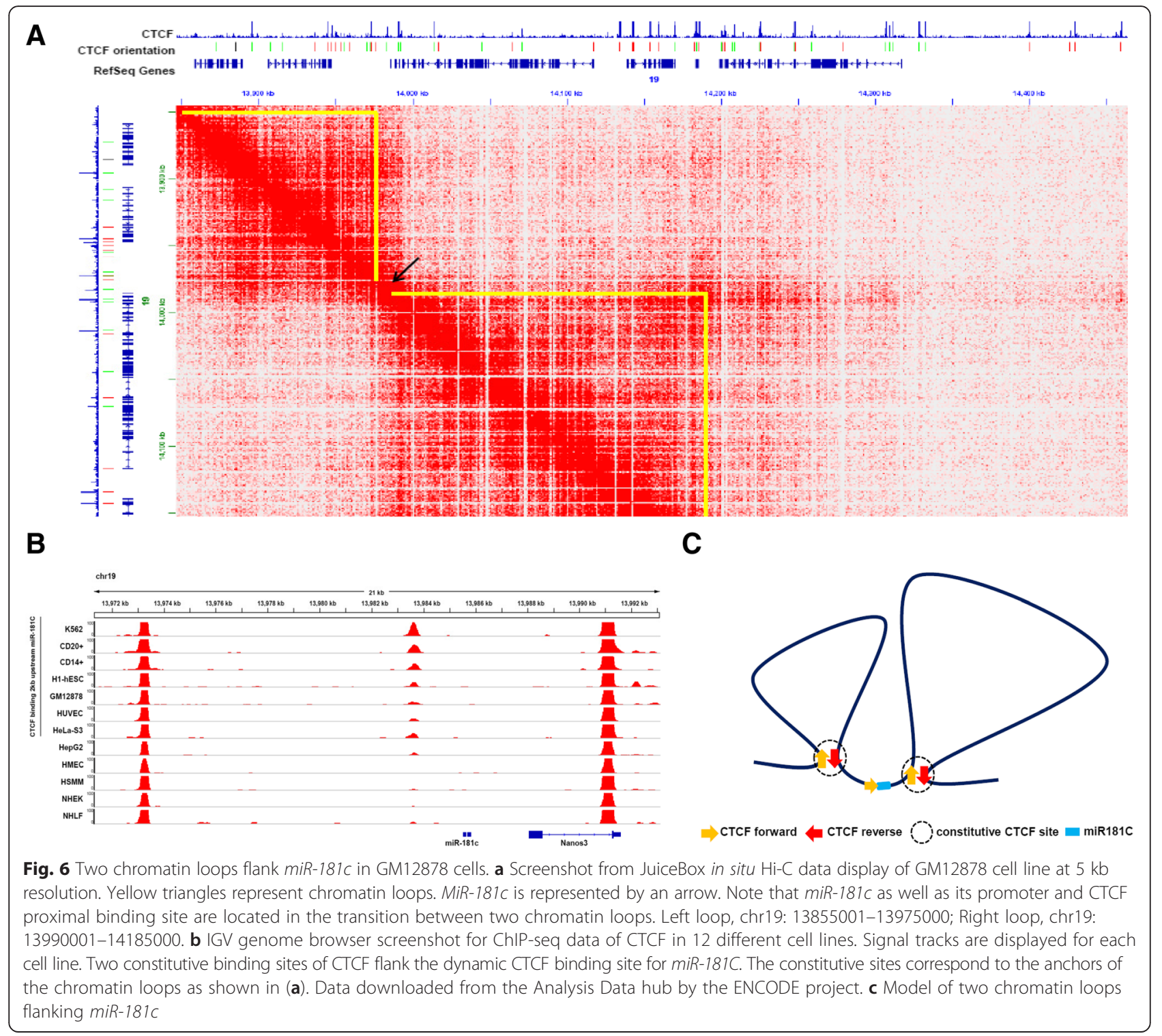

cells [30]. Interestingly, alternative epigenetic silencing mechanisms exist, like the overexpression of EZH2, a key member of the Repressive Polycomb Complex, $\mathrm{PRC} 2$, that in addition to silence many genes, including tumour suppressor genes, can also silence different microRNAs in cancer cells [46]. It has been documented by several research groups that EZH2 is overexpressed in different cancers, and found to repress abnormally different microRNAs, including the miR-181c in prostate and breast cancer cells [47]. Then, based on our observation and the differential binding of CTCF to the miR181c in different cell-types we propose that EZH2 and Polycomb proteins may be responsible for silencing the miR-181c in cell-types were the miR-181c is normally not expressed, like in the human erythroleukemic K562 cells or primary lymphocytes (Fig. 1).
An important aspect that is to a certain extent underestimated is the possibility that in glioblastoma cells CTCF is affected by mutations. Nowadays, there is a repertoire of different CTCF mutations, comprising somatic mutations, resulting in nonsense, missense, frameshift and splice site mutations [48]. Some of these mutations have been identified in different cancer types. From a functional point of view, a large proportion of mutations are found in the zinc-fingers that are critical for CTCF binding to DNA $[48,49]$. Therefore, in glioblastoma cells and in regulatory regions as for the miR-181c, CTCF disruption can be caused by specific mutations that affect its binding to DNA. This view is further supported by a recent report in which ctcf hemizygous knockout mice predisposes to cancer, under certain inducible conditions, promoting tumour 


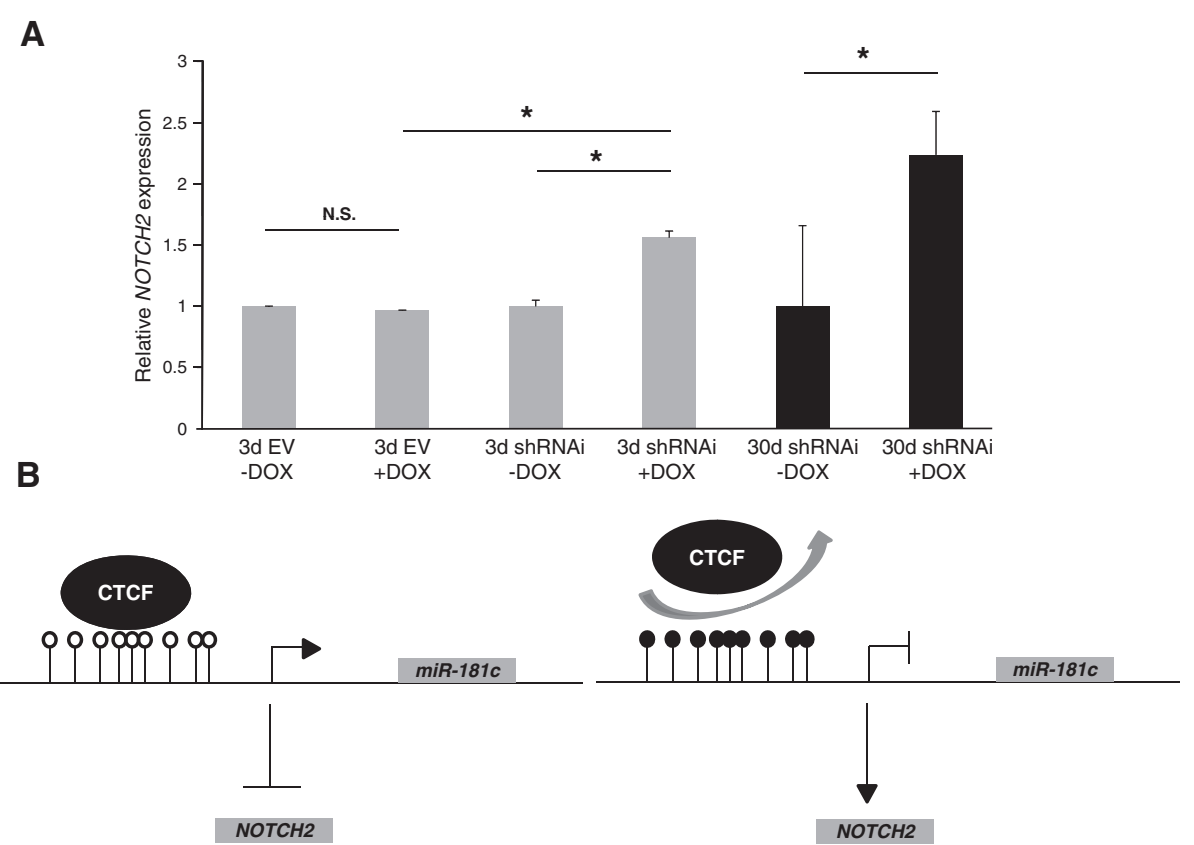

Fig. 7 Depletion of CTCF in U87MG cells affects the transcription levels of NOTCH2. a NOTCH2 mRNA levels in U87MG cells transduced with an inducible shRNAi against CTCF after 3 and 30 days with or without DOX induction (3d shRNAi-DOX; 3d shRNAi + DOX; 30d shRNAi-DOX; 30d shRNAi + DOX). Empty vector controls are shown (3d EV-DOX and 3d EV + DOX). ${ }^{*} p$-value $<0.05$. N.S., not significant. This set of data is representative of three-independent experiments. $\mathbf{b}$ In U87MG cells CTCF binds to the promoter region of miR-181c (Left). Loss of CTCF causes epigenetic silencing of miR-181c by DNA methylation and an increase of NOTCH2 mRNA (Right)

aggressive invasion and metastatic dissemination [50]. What is even more relevant, in the context of the present study, is the fact that CTCF haploinsufficient mice destabilize genome-wide DNA methylation patterns supporting the relationship between CTCF and DNA methylation in certain genomic regions [50]. In the same study point mutations have been correlated with abnormal gain of DNA methylation. Therefore, CTCF is now considered as a tumour suppressor gene in human cancers and is significantly mutated gene in different types of cancers [50, 51].

Based on the recent series of publications and given the architectural role attributed to CTCF we cannot discard, that the CTCF located in the promoter region of the miR-181c plays a structural role [52]. Due to this possibility we analyzed the genomic distribution of CTCF, and its relationship with the three-dimensional architecture of the genome taking advantage of the newly, high resolution, genome-wide mapping of chromatin loops by in situ $\mathrm{Hi}-\mathrm{C}$ [43]. In situ $\mathrm{Hi}-\mathrm{C}$ series of experiments have reached up to $1 \mathrm{~kb}$ resolution. As shown in Fig. 6, the CTCF site associated with the miR181c promoter does not seem to correspond to a loop anchor site (Fig. 6c). We believe that this is relevant, and we propose that this CTCF site is not a structural one, instead we suggest a local regulatory function, in particular, protection against DNA methylation. In addition, Lieberman Aiden and collaborators demonstrated that more than $90 \%$ of the CTCF sites at loop anchors, at the DNA binding sequence level, are positioned in a convergent orientation [43]. This is extremely relevant since this type of sequence convergence orientation for CTCF binding sites turns out to be an excellent predictor of chromatin loop formation. Based in such prediction we propose a model in which the miR-181c, and its adjacent gene Nanos3, are not included in a loop and their location correspond to a genomic region between two large chromosomal loops (Fig. 6c).

In glioblastoma the Notch signaling pathway is aberrantly activated [53]. NOTCH2 is one of the receptors of the Notch pathway and was recently shown to be important for proliferation, invasion and self-renewal of glioblastoma U87MG cells [34]. The NOTCH2 gene is also a post-transcriptionally target of $m i R-181 \mathrm{c}$ and a negative correlation between NOTCH2 gene expression and $m i R-181 c$ was found in glioblastoma samples [34]. In the present study we observed that CTCF knockdown induces overexpression of $\mathrm{NOTCH} 2$ gene in U87MG glioblastoma cells possibly as a consequence of the epigenetic silencing by DNA methylation of miR-181c (Fig. 7a). This finding highlights the importance of 
CTCF as a regulator of gene expression for tumour suppressor genes. In conclusion, microRNAs are subjected to multiple levels of regulation and there are few examples of how they are regulated transcriptionally, and even fewer how they are deregulated epigenetically. Due to their critical role during animal development it is important to continue exploring how these regulatory genes are controlled by a multitude of mechanisms.

\section{Conclusions}

Cancer is a disease that combines genetic and epigenetic defects, in addition, to an active participation of microRNAs. The biogenesis and ways of action of microRNAs are relatively well known but their transcriptional regulation is an aspect that is poorly understood. Here we show that the $m i R-181 c$ is differentially expressed in glioblastoma cell lines. As seen in some tumour suppressor genes CTCF is found in promoter regions protecting them against epigenetic silencing. The absence of CTCF correlates with gain of DNA methylation and the downregulation of the miR-181c expression. Our results support the epigenetic role of CTCF in the regulation of microRNAs implicated in tumorigenesis.

\section{Additional file}

Additional file 1: Figure S1. CTCF binds to the promoter of miR-181C in different cell lines. Figure S2. In vivo CTCF association in the promoter region of the miR-181c. Figure S3. Quantization of the inducible CTCF knockdown in the U87MG glioblastoma cells. Table S1. CTCF binds to the promoter of miR-181c in different cell lines. (PDF $902 \mathrm{~kb}$ )

\section{Abbreviations \\ 5-azadC: 5-aza-2'-deoxycytidine; CTCF: CCCTC-binding factor; DMR: differential methylation region; DOX: doxycycline; ENCODE: encyclopedia of DNA elements; GBM: glioblastoma multiforme; RT-qPCR: quantitative reverse transcription- polymerase chain reaction; shRNAi: small-hairpin interference RNA.}

\section{Competing interests}

The authors declare that they have no competing interests.

\section{Authors' contributions}

EA-O and FR-T designed the study and wrote the manuscript. EA-O, RA-M, RP-M, EG-B, GG and KM performed the experiments. FR-T, RA-M and EA-O performed the bioinformatic analysis of the corresponding genomic region. All authors read and approved the final version of the manuscript.

\section{Acknowledgements}

We thank Paul Delgado-Olguín for critical reading of the manuscript, Gabriel Cuevas from the Instituto de Química (UNAM) for his constant support, Fernando Suaste-Olmos and Guadalupe Códiz Huerta for technical assistance. Ph.D. fellowships from CONACyT and Dirección General de Estudios de Posgrado-Universidad Nacional Autónoma de México (DGEP) (EA-O; RA-M; RP-M; EG-B). Additional support was provided by the PhD Graduate Program, "Doctorado en Ciencias Biomédicas y Ciencias Bioquímicas" and the Instituto de Fisiología Celular from the Universidad Nacional Autónoma de México. We acknowledge the post-doctoral fellowship from DGAPA-UNAM to KM. This work was supported by the DGAPA-PAPIIT, UNAM (IN209403, IN203811 and IN201114), CONACyT (42653-Q, 128464 and 220503) and Join Partnership Program UNAM-UIUC.
Received: 8 September 2015 Accepted: 10 March 2016

Published online: 16 March 2016

\section{References}

1. Jansson MD, Lund AH. MicroRNA and cancer. Mol Oncol. 2012;6(6):590-610.

2. Suzuki H, Maruyama R, Yamamoto E, Kai M. Epigenetic alteration and microRNA deregulation in cancer. Front Genet. 2013;4:258.

3. Benetatos L, Voulgaris E, Vartholomatos G, Hatzimichael E. Non-coding RNAs and $\mathrm{EZH} 2$ interactions in cancer: long and short tales from the transcriptome. Int J Cancer. 2013;133(2):267-74.

4. Di Leva G, Garofalo M, Croce CM. MicroRNAs in cancer. Ann Rev Pathol Mech Dis. 2014;9:287-314.

5. Denis $\mathrm{H}, \mathrm{Ndlovu}$ MN, Fuks F. Regulation of mammalian DNA methyltransferases: a route to new mechanisms. EMBO Rep. 2011;12(7):647-56.

6. Fabbri M, Garzon R, Cimmino A, Liu Z, Zanesi N, Callegari E, et al. MicroRNA-29 family reverts aberrant methylation in lung cancer by targeting DNA methyltransferase 3A and 3B. Proc Natl Acad Sci U S A. 2007;104(40):15805-10.

7. Garzon R, Liu S, Fabbri M, Liu Z, Heaphy CE, Callegari E, et al. MicroRNA-29b induces global DNA hypomethylation and tumor suppressor gene reexpression in acute myeloid leukemia by targeting directly DNMT3A and 3B and indirectly DNMT1. Blood. 2009;113(25):6411-8.

8. Zhao B, Bian EB, Li J, Li J. New advances of microRNAs in glioma stem cells, with special emphasis on aberrant methylation of microRNAs. J Cell Physiol. 2014:229(9):1141-7.

9. Asuthkar S, Velpula KK, Chetty C, Gorantla B, Rao JS. Epigenetic regulation of miRNA-211 by MMP-9 governs glioma cell apoptosis, chemosensitivity and radiosensitivity. Oncotarget. 2012;3(11):1439-54.

10. Ying Z, Li Y, Wu J, Zhu X, Yang Y, Tian H, et al. Loss of miR-204 expression enhances glioma migration and stem cell-like phenotype. Cancer Res. 2013; 73(2):990-9.

11. Lee HK, Bier A, Cazacu S, Finniss S, Xiang C, Twito H, et al. MicroRNA-145 is downregulated in glial tumors and regulates glioma cell migration by targeting connective tissue growth factor. PLOS ONE. 2013;8(2), e54652.

12. Bier A, Giladi N, Kronfeld N, Lee HK, Cazacu S, Finniss C, et al. MicroRNA-137 is downregulated in glioblastoma and inhibits the stemness of glioma stem cells by targeting RTVP-1. Oncotarget. 2013;4(5):665-76.

13. Lee HK, Finniss S, Cazacu S, Bucris E, Ziv-Av A, Xiang C, et al. Mesenchymal stem cells deliver synthetic microRNA mimics to glioma cells and glioma stem cells and inhibit their cell migration and self-renewal. Oncotarget. 2013:4(2):346-61.

14. Filippova GN, Fagerlie S, Klenova EM, Myers C, Dehner Y, Goodwin G, et al. An exceptionally conserved transcriptional repressor, CTCF, employs different combinations of zinc fingers to bind diverged promoter sequences of avian and mammalian c-myc oncogenes. Mol Cell Biol. 1996;16(6):2802-13.

15. Klenova EM, Nicolas RH, Paterson HF, Carne AF, Heath CM, Goodwin GH, et al. CTCF, a conserved nuclear factor required for optimal transcriptional activity of the chicken c-myc gene, is an 11-Zn-finger protein differentially expressed in multiple forms. Mol Cell Biol. 1993:13(12):7612-24.

16. MacPherson MJ, Beatty LG, Zhou W, Du M, Sadowski PD. The CTCF insulator protein is posttranslationally modified by SUMO. Mol Cell Biol. 2009:29(3):714-25.

17. Yu W, Ginjala V, Pant V, Chernukhin I, Whitehead J, Docquier F, et al. Poly(ADP-ribosyl)ation regulates CTCF-dependent chromatin insulation. Nat Genet. 2004;36(10):1105-10.

18. Phillips JE, Corces VG. CTCF: master weaver of the genome. Cell. 2009:137(7): 1194-211.

19. Dixon JR, Selvaraj S, Yue F, Kim A, Li Y, Shen Y, et al. Topological domains in mammalian genomes identified by analysis of chromatin interactions. Nature. 2012;485(7398):376-80.

20. Zuin J, Dixon JR, van der Reijden MI, Ye Z, Kolovos P, Brouwer RW, et al. Cohesin and CTCF differentially affect chromatin architecture and gene expression in human cells. Proc Natl Acad Sci U S A. 2014;111(3):996-1001.

21. Zlatanova J, Caiafa P. CTCF and its protein partners: divide and rule? J Cell Sci. 2009:122(Pt 9):1275-84.

22. Choi NM, Feeney AJ. CTCF and ncRNA regulate the three-dimensional structure of antigen receptor loci to facilitate $V(D) J$ recombination. Front Immunol. 2014;5:49.

23. Saldaña-Meyer R, González-Buendía E, Guerrero G, Narendra V, Bonasio R, Recillas-Targa F, et al. CTCF regulates the human p53 gene through direct interaction with its natural antisense transcript, Wrap53. Genes Dev 2014: 28(7):723-34. 
24. Recillas-Targa F, De La Rosa-Velázquez IA, Soto-Reyes R, Benítez-Bribiesca L. Epigenetic boundaries of tumour suppressor gene promoters: the CTCF connection and its role in carcinogenesis. J Cell Mol Med. 2006;10(3):554-68.

25. Recillas-Targa F, De La Rosa-Velázquez IA, Soto-Reyes E. Insulation of tumor suppressor genes by the nuclear factor CTCF. Biochem Cell Biol. 2011;89(5): 479-88.

26. Engel N, West AG, Felsenfeld G, Bartolomei MS. Antagonism between DNA hypermethylation and enhancer-blocking activity at the H19 DMD is uncovered by CpG mutations. Nat Genet. 2004;36(8):883-8.

27. Chang J, Zhang B, Heatch H, Galjart N, Wang X, Milbrandt J. Nicotinamide adenine dinucleotide (NAD)-regulated DNA methylation alters CCCTCbinding factor (CTCF)/cohesin binding and transcription at the BDNF locus. Proc Natl Acad Sci U S A. 2010;107(50):21836-41.

28. Wang H, Maurano MT, Qu H, Varley KE, Gertz J, Pauli F, et al. Widespread plasticity in CTCF occupancy linked to DNA methylation. Genome Res. 2012; 22(9):1680-8.

29. Saito $Y$, Saito $H$. Role of CTCF in the regulation of microRNA expression. Front Genet. 2012;3:186.

30. Soto-Reyes E, Recillas-Targa F. Epigenetic regulation of the human p53 gene promoter by the CTCF transcription factor in transformed cell lines. Oncogene. 2010;29(15):2217-27.

31. de Souza Rocha Simonini P, Breiling A, Gupta N, Malekpour M, Youns M, Omranipour R, et al. Epigenetically deregulated microRNA-375 is involved in a positive feedback loop with estrogen receptor alpha in breast cancer cells. Cancer Res. 2010;70(22):9175-84.

32. Tata PR, Tata NR, Kühl M, Sirbu IO. Identification of a novel epigenetic regulatory region within the pluripotency associated microRNA cluster, EEmiRC. Nucleic Acids Res. 2011;39(9):3574-81.

33. Lakomy R, Sana J, Hankeova S, Fadrus P, Kren L, Lzicarova E, et al. MiR-195, miR-196b, miR-181c, miR-21 expression levels and 0-6-methylguanine-DNA methyltransferase methylation status are associated with clinical outcome in glioblastoma patients. Cancer Sci. 2011:102(12):2186-90.

34. Ruan J, Lou S, Dai Q, Mao D, Ji J, Sun X. Tumor suppressor miR-181C attenuates proliferation, invasion and self-renewal abilities in glioblastoma. NeuroReport. 2015;26(2):66-73.

35. Sand M, Skrygan M, Sand D, Georgas D, Hahn SA, Gambichler T, et al. Expression of microRNAs in basal cell carcinoma. Br J Dermatol. 2012;167(4):847-55.

36. Jones KB, Salah Z, Del Mare S, Galasso M, Gaudio E, Nuovo GJ, et al. miRNA signature associate with pathogenesis and progression of osteosarcoma. Cancer Res. 2012:72(7):1865-77.

37. Cui MH, Hou XL, Lei XY, Mu FH, Yang GB, Yue L, et al. Upregulation of microRNA 181c expression in gastric cancer tissues and plasma. Asian Pac J Cancer Prev. 2013;14(5):3063-6.

38. Dávalos-Salas M, Furlan-Magaril M, González-Buendía E, Valdes-Quezada C, Ayala-Ortega E, Recillas-Targa F. Gain of DNA methylation is enhanced in the absence of CTCF at the human retinoblastoma gene promoter. BMC Cancer. 2011;11:232.

39. Gomes NP, Espinosa JM. Gene-specific repression of the p53 target gene PUMA via intergenic CTCF-cohesin binding. Genes Dev. 2010;24(10):1022-34.

40. Ozsolak F, Poling LL, Wang Z, Liu H, Liu XS, Roeder RG, et al. Chromatin structure analyses identify miRNA promoters. Genes Dev. 2008;22(22):3172-83.

41. Cui H, Niemitz EL, Ravenel JD, Onyango P, Brandenburg SA, Lobanenkov W, et al. Loss of imprinting of insulin-like growth factor-ll in Wilms' tumor commonly involves altered methylation but not mutations of CTCF or its binding site. Cancer Res. 2001:61(13):4947-50.

42. Sexton T, Cavalli G. The role of chromatin domains in shaping the functional genome. Cell. 2015;160(6):1049-59.

43. Rao SS, Huntley MH, Durand NC, Stamenova EK, Bochkov ID, Robinsin JT, et al. A 3D map of the human genome at kilobase resolution reveals principles of chromatin looping. Cell. 2014;159(7):1665-80.

44. Hanahan D, Feinberg RA. Hallmarks of cancer: the next generation. Cell. 2011;144(5):646-74.

45. Recillas-Targa F. Interdependency between genetic and epigenetic regulatory defects in cancer. Methods Mol Biol. 2014;1165:33-52.

46. Au SL, Wong CC, Lee JM, Fan DN, Tsang FH, Ng IO, et al. Enhancer of zeste homolog 2 epigenetically silences multiple tumor suppressor microRNAs to promote liver cancer metastasis. Hepatalogy. 2012;56(2):622-31.

47. Cao Q, Manu RS, Russo N, Scanlon CS, Tsodikov A, Jing X, et al. Coordinated regulation of polycomb group complexes through microRNAs in cancer. Cancer Cell. 2011:20(2):187-99.
48. Tiffen JC, Bailey CG, Marshall AD, Metierre C, Feng Y, Wang Q, et al. The cancer-testis antigen BORIS phenocopies the tumor suppressor CTCF in normal and neoplastic cells. Int J Cancer. 2013;133(7):1603-14.

49. Nakahashi $H$, Kwon KR, Resch W, Vian L, Dose M, Stavreva D, et al. A genome-map of CTCF multivalency redefines the CTCF code. Cell Rep. 2013; 3(5):1678-89.

50. Kemp CJ, Moore JM, Moser R, Bernard B, Teater M, Smith LE, et al. CTCF haploinsufficiency destabilizes DNA methylation and predisposes to cancer. Cell Rep. 2014;7(4):1020-9.

51. Lawrence MS, Stojanov P, Mermel CH, Robinson JT, Garraway LA, Golub TR, et al. Discovery and saturation analysis of cancer genes across 21 tumour types. Nature. 2014;505(7484):495-501.

52. Ong CT, Corces VG. CTCF: an architectural protein bridging genome topology and function. Nat Rev Genet. 2014;15(4):234-46.

53. Stockhausen MT, Kristoffersen K, Poulsen HS. The functional role of Notch signaling in human gliomas. Neuro Oncol. 2010;12(2):199-211.

\section{Submit your next manuscript to BioMed Central and we will help you at every step:}

- We accept pre-submission inquiries

- Our selector tool helps you to find the most relevant journal

- We provide round the clock customer support

- Convenient online submission

- Thorough peer review

- Inclusion in PubMed and all major indexing services

- Maximum visibility for your research

Submit your manuscript at www.biomedcentral.com/submit

) Biomed Central 